\title{
A Newly Developed PC controlled 200kV FE-TEM
}

\author{
K. Okamoto*, Y. Ohkura*, M. Naruse*, K. Hasegawa*, S. Deguchi*, M. Kawazoe* and M. Kersker** \\ * JEOL Ltd., 1-2, Musashino 3-chome, Akishima, Tokyo, 196-8558 JAPAN \\ ** JEOL USA Inc., 11 Dearborn Road, Peabody, MA 01960, USA
}

Recently, the electron microscope is highly regarded as a main tool as not only a high-resolution imaging equipment but also a total analysis system equipment. In order to achieve more flexible functions in the system, the JEM-2100F electron microscope that can be controlled by PC operation has been newly developed. The JEM-2100F has a $200 \mathrm{kV}$ Schottky type of field emission electron gun, an flexible electron optics system which can operate at various observation modes similar to the JEM-2010F. The JEM-2010F is also equipped with a newly designed side-entry goniometer and an anti-vibration system. Figure 1 shows the external view of the JEM-2100F equipped with an EDS detector, and the specifications of this microscope are shown in Table 1. The main features of the JEM-2100F are as follows.

1. Secure control system: The electron optics system for lenses and deflectors, the vacuum control system, and the aperture control system are controlled by the main CPU directly, and the HT system, the Goniometer control system, and the operation panel system are controlled through the subsystem connected to the main CPU (Fig. 2). Changing and/or setting of the observation conditions can be performed on the movable operation panel and the GUI on the host PC connected to the main CPU through the Ethernet. Thus, since the host PC is independent of the main CPU, even if the host PC hangs up, the TEM itself is not influenced by the hang up, but keeping proper operation.

2. Selectable anti-vibration system: The passive or active air mount system can be installed on the main frame of the microscope as the anti-vibration system. Because the effective system that adapts to the floor vibration condition can be selected, it is easy to get high-resolution images routinely.

3. Ease of specimen control: As the piezo device is incorporated in the newly designed side-entry goniometer, it is very easy to control the specimen position precisely even in the high magnification range. The PC can also control all axes of the specimen drive. The combination of these performances and the specimen high tilt performance of the cryo-configuration (Table 1) make it easy to acquire a series of data on cryotomography, for example.

4. Digital STEM performance: The digital STEM imaging device which is also newly developed has a powerful performance for the direct observation of atomic resolution images, including a Si-dumbbell structure, at the maximum magnification of $50 \mathrm{Mx}$ on the standard monitor of the microscope (Fig.3). It is very useful to analyze atomic scale structures.

5. Analytical performance: The solid angle of the EDS detector is a very important factor for efficient analysis. A newly developed $50 \mathrm{~mm}^{2}$ detector realizes $0.22 \mathrm{sr}$ of a solid angle for the UHR configuration of the JEM-2100F. This performance shows a good for nano-area analysis and elemental mapping. 
Table 1 Main Specifications of JEM-2100F

\begin{tabular}{|l|c|c|c|c|}
\hline \multicolumn{2}{|c|}{ Configuration } & UHR & HR & CRYO \\
\hline \hline \multicolumn{2}{|l|}{ Polepiece } & URP & HRP & CRP \\
\hline \multicolumn{2}{|l|}{ Point Resolution (TEM) } & $0.19 \mathrm{~nm}$ & $0.23 \mathrm{~nm}$ & $0.27 \mathrm{~nm}$ \\
\hline \multirow{2}{*}{$\begin{array}{l}\text { Lattice } \\
\text { Resolution }\end{array}$} & TEM & $0.1 \mathrm{~nm}$ & $0.1 \mathrm{~nm}$ & $0.14 \mathrm{~nm}$ \\
\cline { 2 - 5 } & STEM & $0.2 \mathrm{~nm}$ & $0.2 \mathrm{~nm}$ & - \\
\hline \multicolumn{2}{|l|}{ Specimen tilting angle } & $25^{\circ}$ & $35^{\circ}$ & $70^{\circ}$ \\
\hline \multicolumn{2}{l|}{ EDS solid angle } & $0.22 \mathrm{sr}$ & $0.22 \mathrm{sr}$ & - \\
\hline
\end{tabular}

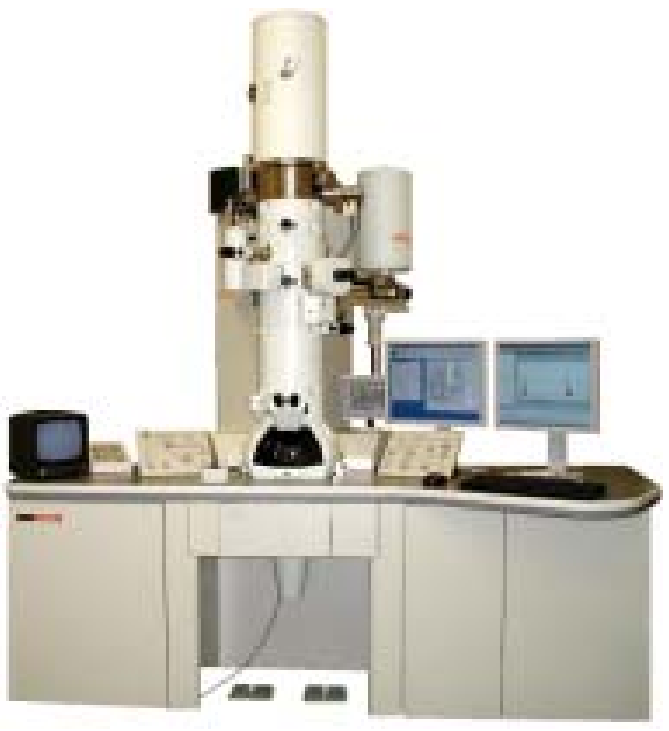

Fig. 1 External view of JEM-2100F.

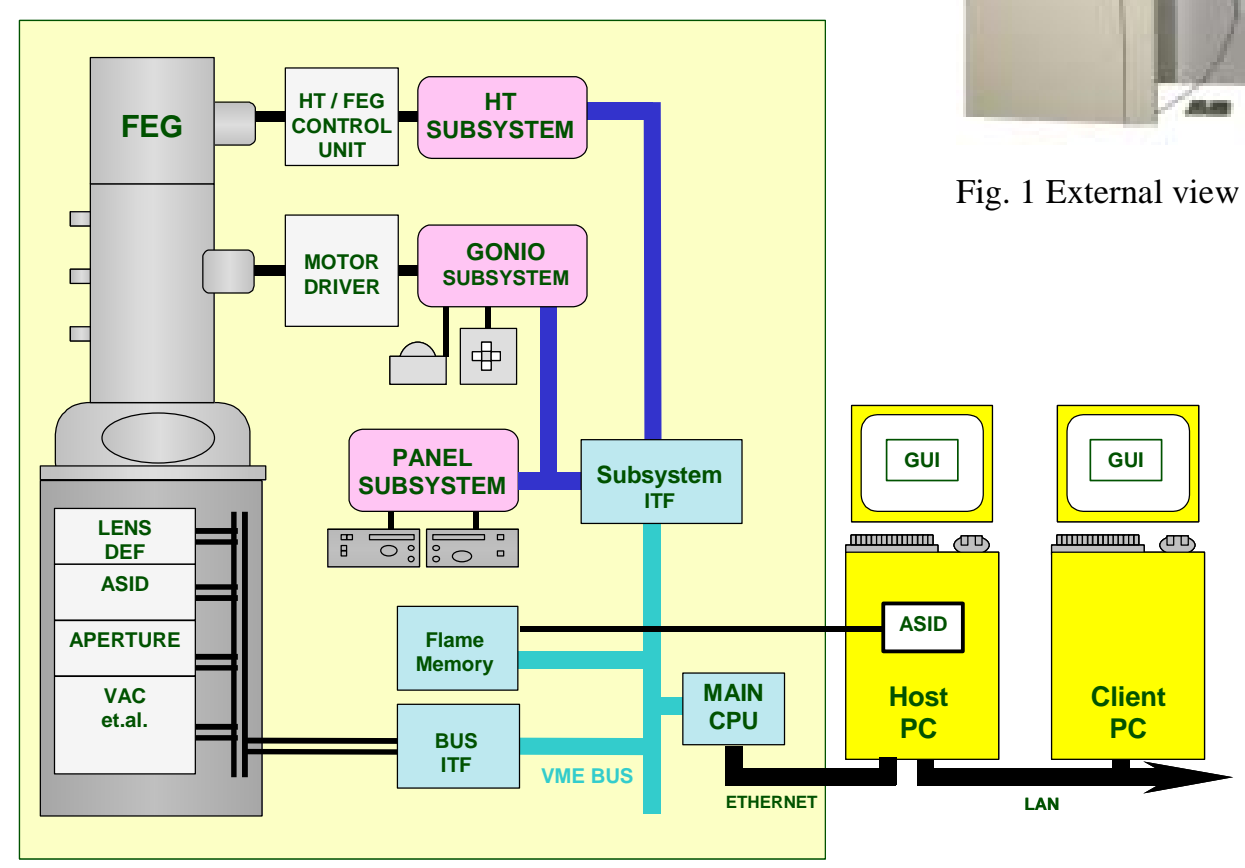

Fig. 2 Block Diagram of JEM-2100F control system

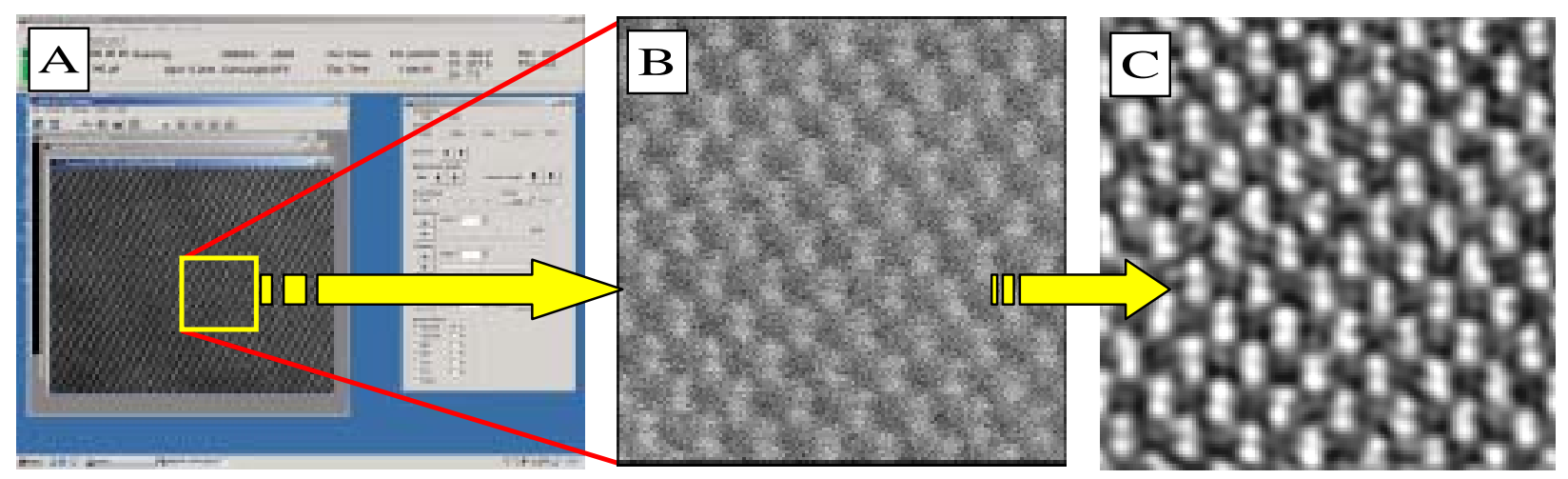

Fig. 3 High resolution STEM HAADF image of Si(110)-dumbbell. A:HAADF image displayed in the GUI window, B: Enlarged image, and C: Filtering Processed image. 